\title{
Éditer des états textuels variants
}

\section{Bénédicte Vauthier}

\section{OpenEdition}

\section{Journals}

Édition électronique

URL : https://journals.openedition.org/genesis/1742

DOI : 10.4000/genesis. 1742

ISSN : 2268-1590

\section{Éditeur :}

Presses universitaires de Paris Sorbonne (PUPS), Société internationale de génétique artistique littéraire et scientifique (SIGALES)

\section{Édition imprimée}

Date de publication : 9 mai 2017

Pagination : $39-55$

ISBN : 979-1023-105636

ISSN : 1167-5101

\section{Référence électronique}

Bénédicte Vauthier, «Éditer des états textuels variants », Genesis [En ligne], 44 | 2017, mis en ligne le 15 mai 2018, consulté le 22 mars 2023. URL : http://journals.openedition.org/genesis/1742 ; DOI : https://doi.org/10.4000/genesis.1742 


\title{
Éditer des états textuels variants
}

\author{
Bénédicte Vauthier
}

\begin{abstract}
Forms effect meaning. Donald F. MCKENZIE

Eine «Fassung» liegt nicht vor, es gibt sie nicht einfach. $\mathrm{Zu}$ einer «Fassung 》 von etwas wird ein vorliegendes Stück Sprache überhaupt erst durch einen Editor. «Fassung ist also ein deskriptiver Begriff, keiner, der etwas so bestimmt, wie es von sich selbst her ist.

Roland REUß
\end{abstract}

$\mathrm{C}$

omme le rappelait récemment encore Almuth Grésillon, dans une entrée «Critique génétique » appelée à figurer dans un «Dictionnaire de philologie éditoriale» de langue allemande («Wörterbuch der Editionsphilologie»), la «critique génétique envisage l'œuvre littéraire comme processus d'écriture; [...] en conséquence, son objet n'est pas le texte édité et clos, mais la diversité et la multiplicité des traces de ce processus, qui, comme des témoins muets, nous sont livrées dans les fonds d'archives littéraires, notamment des auteurs modernes $[\ldots]^{1} »$.

La «génétique éditoriale», quant à elle, ne s'intéresse pas au magma bouillonnant de la création, mais à une phase postérieure du processus d'écriture dans laquelle le «scripteur compose avec les contraintes diverses [...] de l'entrée de l'objet écrit d'abord dans le cercle restreint des acteurs de l'édition (phase pré-éditoriale), puis dans le circuit plus large des acteurs de la réception (phase post-éditoriale)» (voir la présentation du numéro, p. 9). Ce sont en particulier les «transformations effectuées par l'auteur après la mise en circulation du texte ${ }^{2}$ » qui ont retenu l'attention des participants du séminaire «Manuscrit - Linguistique - Cognition », consacré durant trois ans à cette question (2012-2015), à l'initiative de Rudolf Mahrer. La génétique éditoriale se donne ainsi pour héritière de la critique génétique - elle est une «étude des processus »-, mais elle vient prendre le relais d'une tâche que cette approche semblait avoir en partie abandonnée aux philologues.

L'idée d'un continuum qui mènerait un projet d'écriture de l'atelier de l'écrivain aux mains du lecteur, en passant par celles d'une «instance éditeur (editor ${ }^{3}$ )», s'enracine chez Mahrer

1. Almuth Grésillon, «"Critique génétique": Probeartikel», Beihefte zu Editio, «Editorische Begrifflichkeit. Überlegungen und Materialen zu einem "Wörterbuch der Editionsphilologie"», n 36, 2013, p. 197; je traduis.

2. Jean-Louis Lebrave, «Manuscrits de travail et linguistique de la production écrite», Modèles linguistiques, t. XXX, vol. 59, 2009, p. 18.

3. Par «instance "éditeur" ( (de l'anglais editor), je me réfère au caractère collaboratif de la production. Cette instance englobe différentes fonctions «éditoriales» (maquettiste, éditeur scientifique, éditeur responsable, etc.). Voir John Bryant, The Fluid Text. A Theory of Revision and Editing for Book and Screen, Ann Arbor, University of Michigan Press, 2002, p. 7; Peter Shillingbsurg, «Authority and Authorization in American Editing», Beihefte zu Editio, $\mathrm{n}^{\circ}$ 21, 2004, p. 73-81, en particulier p. 77 sq.; Alberto Cadioli, Le diverse pagine. Il testo letterario tra scrittore, editore, lettore, Milano, Il Saggiatore, 2012; Paola Italia, Editing Novecento, Roma, Salerno, 2013. 
dans sa pratique éditoriale des Euvres complètes de Ramuz (2007)4; et elle a trouvé une caution théorique dans une récente contribution de Jean-Louis Lebrave qui a proposé de revoir l'opposition tranchée entre critique génétique et philologie sur la base d'un examen des types de variations présentes dans les brouillons, d'une part, dans des états textuels, d'autre part.

Fidèle à la tradition, c'est la publication qui marque la frontière des phases 3 et 4 dans le schéma de Lebrave (voir p. 9) que Mahrer a retenue comme pivot de la génétique éditoriale5. Toutefois Mahrer souhaite repenser les frontières entre processus et produit en soulignant le caractère provisoire du bon à tirer ou de la publication : il propose de «considérer la publication, non plus comme le terme des processus d'écriture, mais comme un acte et un objet transformant les conditions de la réécriture » (p. 13). Il inscrit ce déplacement dans le sillage des travaux de Donald F. McKenzie, qui a notamment mis en évidence le caractère social de la figure auctoriale, de Cesare Segre, qui a souligné la complémentarité existant entre la critique génétique et la critica delle varianti, étant donné que celle-ci s'intéresse tout particulièrement aux « retouches visant à améliorer le texte achevé 6 », ou encore d'Anne Réach-Ngô, qui a étudié les «genèses éditoriales » à la Renaissance?.

De mon côté, j'ai également fait mienne la réflexion de Lebrave dès 2009, soit au moment d'entreprendre l'étude du dossier génétique puis l'édition (critique, dans un volume imprimé, génétique, sur un portail numérique) de Paysages après la bataille (1982; trad. fr. 1985), roman de l'écrivain espagnol Juan Goytisolo ${ }^{8}$. Je cherchais alors à «(ré)concilier critique génétique et philologie ${ }^{9} »$, ce qui semblait envisageable au départ d'une compréhension de la première comme «poétique des transitions entre états 10 ».

À la suite des trois années d'échanges sur la génétique éditoriale11 et d'une réflexion parallèle liée à l'étude et à l'édition du dossier génétique complexe de La media noche. Visión estelar de

4. Cette même expérience pratique d'un texte fluide («The Fluid Text Moment») est à l'origine de la théorie de J. Bryant (op. cit., 64 sq.).

5. Voir sa contribution dans ce volume, ainsi que l'article collectif Rudolf Mahrer et al, «Editorial genesis : from comparing texts (product) to interpreting rewritings (process)», dans Writing(s) at the Crossroads : The processproduct interface, dir. Georgeta Cislaru, Amsterdam, Benjamins Publishing Company, 2015, p. 151-170, en particulier p. $152-154$.

6. Cesare Segre, «Critique des variantes et critique génétique», Genesis, n 7, 1995, p. 30.

7. Anne Réach-Ngô, «Peut-on parler de genèse éditoriale au XVIe siècle ?», Seizième Siècle, « Genèses éditoriales », $\mathrm{n}^{\circ} 10$, «Présentation», 2014, p. 7-14

8. Bénédicte Vauthier (éd.), Juan Goytisolo, Paisajes después de la batalla. Preliminares y estudio de crítica genética de Bénédicte Vauthier, Salamanca, Ediciones Universidad Salamanca, 2012; B. Vauthier, "Géographie de l'exil de Juan Goytisolo : sur les traces génétiques et intertextuelles de Paisajes después de la batalla », Genesis, ${ }^{\circ}$ 35, 2012 , p. 221-234; Manuscrito digital Juan Goytisolo, 2013-2014. En ligne : < www.goytisolo.unibe.ch >.

9. J.-L. Lebrave, art. cité, p. 18.

10. Ibid., p. 19. C'est dans cette perspective qu'a été organisé le second «Congreso Internacional de manuscritos literarios » (San Millán de la Cogolla, 2009). Le sous-titre du volume qui rassemble les travaux issus de cette rencontre, réunissant pour la première fois en Espagne des généticiens français et des hispanistes, fait état de cette décision : Crítica genética y edición de manuscritos hispánicos contemporáneos. Aportaciones a una "poética de transición entre estados ». Le syntagme figure également dans le titre de mon introduction à l'édition de Juan Goytisolo : «Paisajes después de la batalla a la luz de una "poética de transiciones entre estados"».

11. Si, d'un point de vue institutionnel, j'ai coorganisé et animé le séminaire avec Rudolf Mahrer (voir programme en ligne sur le site de l'ITEM), c'est bien lui qui en a été le capitaine et le gouvernail. Qu'il soit remercié ici pour ses stimulantes réflexions. 
un momento de guerra (1917) de Ramón del Valle-Inclán ${ }^{12}$, je souhaiterais, dans le cadre de cette contribution, revenir sur mon édition de Juan Goytisolo et examiner quelques problèmes théoriques que soulève la pratique d'éditeur scientifique quand elle aborde le texte dans sa dimension processuelle. Il en va ainsi du choix de l'édition (quel état textuel privilégier?), de l'auctorialité multiple (qui corrige ?), des modes d'intervention éditoriale mais aussi et surtout des rapports entre version et texte dans la phase éditoriale (qu'éditer? que représenter et comment?).

Dans un premier temps et afin de donner suite au second enseignement cardinal de D.F. McKenzie qui s'intéresse également au livre en tant qu'objet physique et historique - car «Forms effect meaning $13 »-$, je commencerai par présenter quelques caractéristiques «matérielles» ou «bibliographiques» des huit éditions du roman, publiées avec le consentement, tacite ou explicite, de l'auteur, complément indispensable des caractéristiques «textuelles » des mêmes éditions. J'exposerai ensuite les choix éditoriaux d'un travail qui n'a pu bénéficier à l'époque de la réflexion sur «le texte variant» et reste, en ce sens, prisonnier des principes de la «critique textuelle», d'application pour le texte ancien. Poursuivant une recherche de caractère comparatiste, je chercherai enfin à voir comment la génétique éditoriale française pourrait entrer en dialogue avec les traditions philologiques européennes du «texte moderne» qui font une place à l'étude des «textes variants », problème que j'ai identifié ailleurs comme étant celui des «redazioni plurime ${ }^{14} »$. Il en irait ainsi de la Filologia d'autore italienne, de la Neuphilologie allemande ou de la New New bibliography 15 anglo-américaine, trois approches qui n'ont à l'heure actuelle aucun équivalent dans le domaine hispanique ${ }^{16}$.

Ceci étant dit, et afin d'éviter tout malentendu, je me dois de préciser l'empan de cette réflexion comparatiste, en soulignant que les différentes réflexions d'éditeurs et de philologues que j'évoquerai ici ne prétendent pas être représentatives «d'une philologie17 ». Dans le cadre d'un article, et en l'absence de toute traduction en français de textes lus en italien, en allemand,

12. B. Vauthier, «¿Crítica textual? ¿Critique génétique? ¿Filologia d’autore? ¿Crítica genética? Edición de manuscritos hispánicos contemporaréanos e hispanismo (inter) nacional», dans Juan Ramón Jiménez y los borradores inéditos de sus archivos. Nuevas propuestas metodológicas, dir. Teresa Gómez Trueba, Valencia, Renacimiento, 2014, p. 22-70; « ¿Critique génétique y/o filologia d'autore? Según los casos... "Historia” — ¿o fin? — "de una utopía real" », Creneida. Anuario de literaturas hispánicas, 2 ; disponible en ligne : < www.creneida.com/revista/ creneida-2-2014/ > ; B. Vauthier et Margarita Santos Zas, «La media noche. Visión estelar de un momento de guerra (1917) de Valle-Inclán. Génesis de un relato escrito desde la vanguardia », Romanische Forschungen, ${ }^{\circ}$ 127, 2015, p. 328-348; ibid. (éd.), Un día de guerra (Visión estelar). La Media Noche. Visión estelar de un momento de guerra de Rámon del Valle-Inclán. Estudio y dossier genético y editorial, Santiago de Compostela, Universidade de Santiago, 2017.

13. Roger Chartier, «Textes, formes, interprétations », préface à Donald F. McKenzie, La Bibliographie et la sociologie des textes, Paris, éd. Cercle [original anglais 1985], 1991, p. 7.

14. «¿Critique génétique y/o filologia d'autore? Según los casos... », art. cité.

15. J'emprunte l'étiquette, courante parmi les anglicistes, à Geert Lernout. Le premier adjectif renvoie à l'idée du «changement de paradigme» qui s'opère parmi les textualistes anglo-américains, qui, au début des années quatrevingt, cherchent à établir les bases d'une approche du texte moderne (voir aussi Helge Nowak, «Umbruch-Zeiten. Paradigmenwechsel innerhalb der anglo-amerikanischen Editionwissenschaft», Editio, n 10, 1996, p. 1-24).

16. B. Vauthier et M. Santos Zas (éd.), op. cit. ; B. Vauthier, «Historia de las lecturas de McKenzie. De la Bibliography a la Histoire du livre, con un alegato a favor de una sociología de los textos lato sensu», Revista Hipánica Moderna, 2017 (sous presse).

17. Ici, comme ailleurs, il me faut toutefois prendre des précautions oratoires et préciser qu'il n'existe pas «une» philologie, pas plus qu'il n’existe «une» critique génétique. 
en anglais, je suis consciente du caractère quelque peu lapidaire qu'aura ma démarche. Celui qui connaît un peu les traditions étrangères trouvera peut-être mon esquisse trop rapide, mais j'ose espérer que celui qui les ignore y trouvera une raison suffisante pour avoir envie de s'y confronter. Enfin, s'il me semble impératif de situer la génétique éditoriale par rapport à la critique génétique, c'est uniquement dans le but de voir si celle-là peut aller à la rencontre des philologues du texte moderne qui cherchent une manière de «réconcilier critique génétique et philologie» respectueuse des spécificités de leur objet : l'avant-texte, d'une part, le texte, d'autre part.

\section{Retour sur un «dossier philologique». Éditer la genèse éditoriale}

Sans compter mon édition parue à Salamanque en 2012, il existe huit «éditions» en espagnol de Paysages après la bataille.

1. Paisajes después de la batalla, Barcelone, Montesinos, 1982. Il s'agit de l'édition princeps. Une photo d'Alicia Liddell, que l'on doit à l'auteur d'Alice au pays des merveilles, illustre la couverture. Les pans intérieurs de celle-ci forment, une fois joints, un plan du métro de Paris.

2. Paisajes después de la batalla, Barcelone, Edicions del Mall, 1985 (format $16 \times 21 \mathrm{~cm}$ ). Il s'agit d'une édition illustrée par Eduardo Arroyo. L'édition est également accompagnée d'un épilogue de Julián Ríos, directeur de la collection et ami de l'auteur et de l'illustrateur.

3. Paisajes después de la batalla, Barcelone, Círculo de Lectores, 1987. D'un nouveau format $(14 \times 19 \mathrm{~cm})$ qui affecte la mise en page, l'édition réintroduit les illustrations d'Eduardo Arroyo, et comprend une introduction de Jesús García Gabaldón et une réflexion finale de Robert Kiely. C'est de cette édition que l'auteur est reparti pour établir l'«édition d'auteur» de 2006.

4. Paisajes después de la batalla, Madrid, Espasa-Calpe, 1990. Il s'agit de la première édition au format de poche dans une collection qui s'adresse à un large public. Andrés Sánchez Robayna, écrivain, critique de grande renommée, et ami personnel de l'auteur, repart de l'édition de 1987, qu'il considère comme la «plus soignée des trois», et déclare qu'elle aurait été «revue par l'auteur» (1990, p. 38). Son édition est la seule qui soit assortie d'une courte «note éditoriale » qui fait état de légères modifications apportées au texte et affectant l'orthographe et la ponctuation. Elle inclut aussi quelques notes de contenu, mais fait l'économie des illustrations.

5. En 1999, Espasa-Calpe offre une «réimpression» $\mathrm{du}$ «texte» dans une collection d'ouvrages emblématiques du $\mathrm{XX}^{\mathrm{e}}$ siècle en langue espagnole. Le livre est toutefois publié sans l'introduction et la note éditoriale de Sánchez Robayna.

6. Paisajes después de la batalla, Obras completas III. Novelas (1966-1982), Barcelone, Galaxia Gutenberg/Círculo de lectores, 2006, p. 881-1047. Entre 2006 et 2007, Juan Goytisolo a été associé aux travaux d'édition de ses œuvres complètes. Il est stipulé sur la couverture et sur la page de titre de chacun des volumes qu'il s'agit d'une «édition de l'auteur, à charge d'Antoni Munné». Goytisolo signale dans le prologue que le roman a été modifié : une séquence («Elle») a été supprimée pour des raisons «autobiographiques» et la fin de la séquence précédente ( Sa vie est un songe») a été partiellement réécrite pour des raisons de cohésion textuelle. En outre, l'auteur a retouché la graphie de certains mots, changé l'un ou l'autre temps verbal, revu la ponctuation. On peut prendre connaissance de ces modifications en consultant l'exemplaire de l'édition de 1987 qui a servi au travail de réédition du «texte» (ses illustrations n'ayant pas été réincorporées). De fait, Antoni Munné m'avait expliqué comment il travaillait avec l'auteur : celui-ci a relu dans une édition de son choix tous les textes à rééditer, annotant dans les marges les modifications à apporter. Munné préparait alors la nouvelle édition en amendant le texte de référence selon les instructions données. Il lui serait arrivé de suggérer de légères «améliorations» concernant, par exemple, la ponctuation, l'orthographe. Mais ces questions, avait-il précisé à mon plus grand étonnement, se réglaient généralement par téléphone - Juan Goytisolo vit à Marrakech, et son éditeur à Barcelone - et, donc, sans laisser de traces.

7. En 2006, cette même «version» du texte a été reproduite dans un volume qui comprend trois romans de l'auteur des années 1980-1990, rassemblés sous le titre Profecías, asedios y apocalipsis (Barcelona, Aleph). Aucune 
note n'accompagne cette réédition qui inclut pourtant les modifications de l'édition précédente, auxquelles s'ajoute l'une ou l'autre nouvelle «variante» orthographique.

8. En 2013, les éditeurs Galaxia Gutenberg/Círculo de Lectores font paraître une édition isolée de Paisajes después de la batalla. Le texte suit le modèle de 2006 sans qu' aucune note ne vienne le préciser.

Voilà pour une très brève présentation des éditions disponibles et l'essentiel de leurs caractéristiques matérielles. Voyons maintenant ce qu'il en est du «texte».

En 2012, forte d'une habitude éditoriale bien établie, j'ai respecté la «dernière » volonté de l'auteur et j'ai réédité sans exception aucune - en réalité à une exception près - ce que je pensais être le nouveau «texte de référence », à savoir un roman composé de soixante-seize séquences, au lieu des soixante-dix-sept initiales. À la fin du livre, en appendice, j'ai reproduit le fragment de l'édition de 1982 supprimé en 2006. Et j'ai signalé la modification de la séquence précédente dans une courte note de bas de page.

J'ai construit un apparat critique en bas de page dans lequel, à l'exclusion de la ponctuation, étaient indiquées dans l'ordre chronologique toutes les modifications observées. En voici, sous forme condensée, un bref échantillon :

\begin{tabular}{|l|l|}
\hline \multicolumn{1}{|c|}{ Texte } & Note \\
\hline «... no era producto de la casualidad sino que llevaba*, por así decirlo» (p. 203) & sino llevaba $(1982,1985)$ \\
\hline «Así lo refirió a sus colegas al* día siguiente» (p. 204) & el (1982, 1985) \\
\hline « sino letras de esas*», p. 204 & ésas $(1982,1985)$ \\
\hline «estaba seguro de que eran idénticos*»,p. 204 & iguales $(1982,1985)$ \\
\hline «habían sido remplazados por* signos», p. 206 & con (1982, 1985, 1987, 1990) \\
\hline «L'humanité*», p. 206 & L'humanité (1982, 1985, 1987) \\
\hline «paquistanís*», p. 207 & paquistaneses (1982, 1985, 1987), paquistanses (sic, 1990) \\
\hline
\end{tabular}

Il s'agit, on le voit, de corrections minimes et très circonscrites (généralement un mot) : élimination de possibles maladresses stylistiques (changement de préposition), voire d'erreurs ( «sino que» versus «sino», «al día» versus «el día», etc.). On constate aussi des adaptations orthographiques aux normes en vigueur de l'Académie ( «ésas» versus «esas», «mare mágnum» ou «maremágnum»), des rectifications de mots moins usuels («paquistaneses» versus «paquistanís», «seudohippie» pour «seudohippy», dont les deux graphies sont pourtant acceptées) ou, enfin, des corrections typographiques (utilisation de l'italique pour un titre).

Un bilan général de ces micro-réécritures permet de voir que l'édition de 1985 suit rigoureusement le texte de 1982. Un certain nombre de «leçons » ne sont présentes que dans les éditions de 1982 et 1985, avant d'être modifiées - mais par qui ? - en 1987. Sánchez Robayna est fidèle à sa déclaration et suit le texte de 1987 à deux ou trois exceptions près, où il corrige de possibles «erreurs de langue». Quelques coquilles ne sont toutefois présentes que dans cette édition, puis corrigées dans l'édition de l'auteur de 2006. La seconde édition de 2006 (Aleph) fait état de nouvelles divergences ponctuelles, probables corrections du maquettiste. Dans une optique philologique, toutes ces réécritures pouvaient aisément être documentées en note de bas de page.

Mais la mention «édition d'auteur» et le prologue auctorial nous autorisent-ils pour autant à considérer le texte publié, ou plutôt ces différentes réécritures, si minimes soient-elles, comme étant celles d'un seul scripteur? S'agit-il là de réécritures «autorisées » ? Ou seulement d'une «édition autorisée»? Ces questions ne sont pas anodines dans l'optique de la génétique éditoriale.

Pour essayer d'y voir plus clair, arrêtons-nous sur un exemple significatif de réécriture entre A, l'édition de 1987, et B, l'édition de 2006. Ces deux objets constituent «des états différents de ce qu'on peut supposer être un même 
texte 18 ». Par ailleurs, l'édition de 1987 - non celle de Sánchez Robayna de 1990, ni la princeps de 1982 - a servi à l'auteur pour établir le texte de 2006 : la comparaison génère donc bien des variantes génétiquement pertinentes (au sens de Mahrer, p. 33), concrètement elle permet de localiser une substitution («misteriosa» pour «verdadera») et un ajout («sin permitirnos... perdonará!») (tableau-ci-contre).

Jetons maintenant un coup d'œil sur l'exemplaire de l'auteur, qui permet au généticien d'avoir accès au processus de réécriture éditoriale (fig. 1).

$\mathrm{Si}$ on compare cet exemplaire annoté et la version de 2006, on observe deux réécritures supplémentaires - une modernisation orthographique et une injonction à corriger la référence à la page du «manuscrit» cité dans le texte selon la pagination de l'édition en cours («la que corresponde a la edición»). Or ni l'une ni l'autre n'ont été intégrées dans l'édition de 2006. Pourtant la demande de correction du mot «mare mágnum» a été suivie quelques pages avant, engendrant ainsi une incohérence orthographique dans l'édition. Quant au silence qui suit l'invitation faite à adapter la référence interne à la pagination de l'édition en cours, rien ne l'explique.

Que faire face à ces énigmes ? Comment y donner suite non pas dans une étude de genèse éditoriale, mais dans ce qui prétend être une réédition d'un «texte autorisé »? Peut-on, doit-on, suspecter une intervention de l'éditeur sur le texte «définitif» «de» Juan Goytisolo, puisque les indications de ce dernier ne semblent pas avoir été suivies sur ces points? Ou tout simplement un oubli, voire une incompréhension de l'injonction de la part de l'éditeur? Faut-il imaginer que l'auteur a changé d'avis et a fait marche arrière lors de la correction d'épreuves ? Cela paraît hautement invraisemblable dans le cas de l'injonction à corriger et plutôt incohérent dans le cas de «mare mágnum». Peut-on encore imaginer que l'auteur n'a pas relu les épreuves, ni procédé à une vérification des amendements souhaités, donnant ainsi un consentement tacite au travail de l'éditeur qui servira ensuite aux critiques et interprètes du roman?

18. Jean-Gabriel Ganascia, Jean-Louis Lebrave, «Trente ans de traitement informatique des manuscrits de genèse », dans Critique génétique. Concepts, méthodes, outils, dir. O. Anokhina et S. Pétillon, Caen, IMEC éditeur, 2009, p. 74 ; je souligne.
Je n'ai pas de réponse définitive à donner. Mais nous voyons clairement le délicat problème que cet exemple - parmi tant d'autres - soulève, d'une part, au sujet de la «volonté auctoriale» et d'une possible «auctorialité multiple»; d'autre part, au sujet de la pertinence de comparer «des chaînes de caractère 19 » qui ne sont plus nécessairement attribuables à un seul et même scripteur. En effet, dans la phase éditoriale, alors que les processus n'apparaissent souvent qu'au travers de leurs effets sur les textes imprimés, les contours de la «figure auctoriale» sont floutés; on ne peut plus toujours déterminer avec certitude qui, de l'auteur, de l'éditeur ou du typographe, corrige. Sitôt le texte sorti de l'atelier de l'écrivain, il entre, en effet, dans un jeu complexe de négociations dont les fils sont parfois difficiles à démêler. Comme le souligne Donald Reiman, qui creuse une idée avancée par McGann en 1983, reprise par McKenzie en 1985, «vouloir qu'une œuvre soit rendue publique signifie (dans pratiquement tous les cas) vouloir qu'elle subisse le processus de socialisation. Un auteur qui envoie son œuvre à l'imprimerie est conscient du fait qu'elle en ressortira sous une apparence différente de celle du manuscrit 20 ». En outre, les omissions relevées ici, et l'existence d'un exemplaire annoté vont me permettre d'évoquer le danger - téléologique et intentionnaliste - que peut représenter à ce stade la réouverture des frontières entre avant-texte et texte.

Pour ma part, et n'ayant pas eu connaissance de précédents dans le domaine hispanique, j' ai résolu cette question de manière pragmatique - et intuitive - en 2012. Au départ de l'exemplaire annoté, j'ai indiqué en bas de page, toutes les réécritures de 2006, en spécifiant celles qui seraient attribuables «à l'auteur», même si certaines d'entre elles n'ont pas été suivies. Par contre, j'ai pris la liberté de corriger dans le corps du texte (!) le renvoi à la page interne en me prévalant non seulement des indications de l'exemplaire annoté, mais aussi d'un autre indice métadiscursif, présent dans le dossier génétique, qui laissait lui aussi penser qu'il

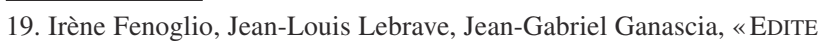
MEDITE : un logiciel de comparaison de versions », ITEM [en ligne], 2007, disponible sur : < www.item.ens.fr/index.php?id=172967>.

20. Donald H. Reiman, The Study of Modern Manuscripts : Public, Confidential and Private, Baltimore/London, John Hopkins University Press, 1993, cité par Geert Lernout, «La critique textuelle angloaméricaine : une étude de cas », Genesis, n 9, 1996, p. 56. 


\section{A = Édition de 1987, p. 266-267}

Un repaso a las doscientas cuarenta y ocho páginas de su manuscrito descubre la existencia de un ser fragmentado : ideas, sentimientos, libido tiran por diferentes caminos, el desdichado cronista de su vida ha sido incapaz de aglutinarlos. Hojear su relato acuciado por la premura del tiempo es un lancinante ejercicio de irrealidad : al final, ya no sabe si es el remoto individuo que usurpa su nombre o ese goytisolo lo está creando a él. Los materiales dispersos en su mesa de trabajo le sumen en un mare mágnum de incertidumbres y, angustiado, abrirá uno de los cajoncillos superiores de aquélla y examinará la fotografía de su verdadera mujer.

\section{B = Édition de 2006, p. 1036}

Un repaso a las doscientas cuarenta y ocho páginas de su manuscrito descubre la existencia de un ser fragmentado : ideas, sentimientos, libido tiran por diferentes caminos, el desdichado cronista de su vida ha sido incapaz de aglutinarlos. Hojear su relato acuciado por la premura del tiempo es un lancinante ejercicio de irrealidad : al final, ya no sabe si es el remoto individuo que usurpa su nombre o ese goytisolo lo está creando a él. Los materiales dispersos en su mesa de trabajo le sumen en un mare mágnum de incertidumbres y, angustiado, abrirá uno de los cajoncillos superiores de aquélla y examinará la fotografía de su misteriosa mujer, sin permitirnos siquiera que le veamos la cara. ¡Una manifestación más de secretismo y desconfianza que el honrado lector no le perdonará !

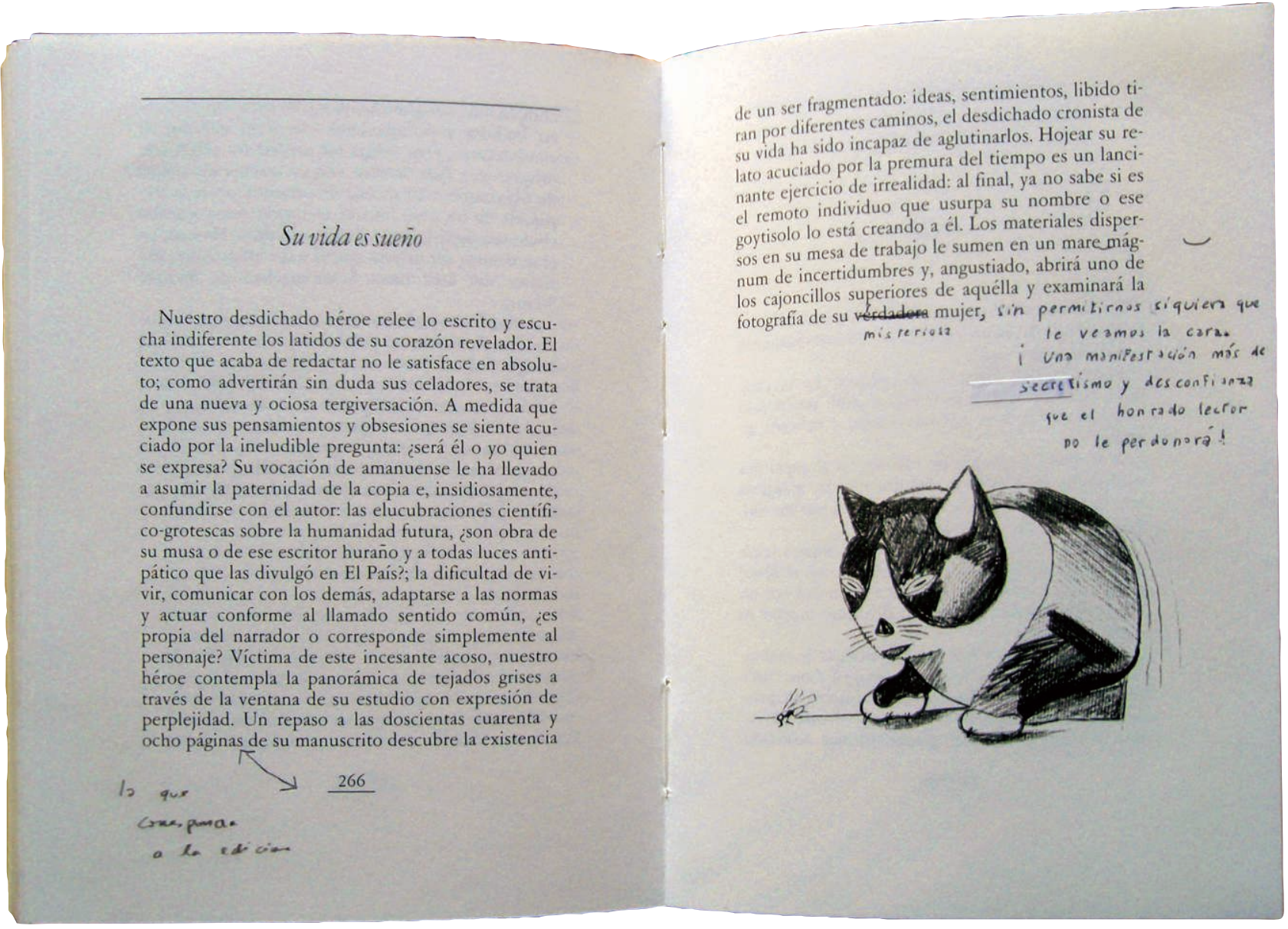

Fig. 1 : Paisajes después de la batalla, Barcelone, Círculo de Lectores, 1987; exemplaire annoté par l'auteur (c) Bénédicte Vauthier 
s'agissait d'un jeu autoréférentiel «voulu par l'auteur». Bien qu' aucune édition n' ait jamais réellement intégré cette information en ajustant cette référence à la pagination du livre, ma décision se basait ainsi sur la connaissance du dossier génétique prééditorial, c'est-à-dire sur l'identification de la seule numération autographe visible sur un feuillet du manuscrit de travail : «171», inscrit dans le coin supérieur droit de la page. En outre, un premier brouillon montrait que l'auteur était passé d'une référence abstraite à la lecture de l'œuvre («La lectura de» «La revisión de su propia obra») à celle de la foliotation du manuscrit («El repaso de» «las 170 páginas de su manuscrito») ${ }^{21}$. D'un point de vue «interprétatif» (Deutung), les réécritures témoignaient d'un jeu métadiscursif de portée métaleptique. Celui-ci semblait corroboré par l'injonction auctoriale de 2006.

Enfin, et pour revenir à notre point de départ, quel était le parti éditorial à adopter face aux réécritures de l'édition «autorisée » de 2006 ?

Alors que, forte des principes de la critique génétique, et pour des raisons de volume, j'avais renoncé à éditer le dossier au format papier, une seule réécriture liée (la suppression d'une séquence, suivie d'un ajout de deux lignes dans la séquence antérieure) semblait «autoriser» l'élaboration d'un apparat, qui s'accommodait bien aux corrections orthographiques, et «légitimer» une présentation texte-apparat plutôt qu'exiger une édition autonome des deux textes.

Mais indépendamment de son caractère isolé, cette réécriture n'était-elle pas le signe d'un «changement de poétique » qui aurait justifié que l'on résolve d'abord la «difficulté épistémologique que soulève ce problème de textes à la fois identiques et différents 22 », et, par conséquent du statut du texte, des textes?

En 2006, Juan Goytisolo explique la suppression de la séquence en signalant que ce qu' «Elle» signifiait pour lui - Monique Lange, la vraie femme, ou la femme mystérieuse dont le personnage ne montrerait finalement pas la photo - il l'avait déjà dit dans des écrits autobiographiques (en 1985,

21. Ces opérations peuvent être observées sur le portail Manuscrito digital de Juan Goytisolo : < www.goytisolo.unibe.ch/capitulos.html> («Su vida es sueño [70]»).

22. J.-G. Ganascia et J.-L. Lebrave, art. cité, p. 74.
1996 et 2003). Ce qui justifiait qu'il fasse l'économie d'une séquence qui brisait l'équilibre romanesque.

Sur la base de cette déclaration, pouvait-on encore réellement penser que nous étions face à «deux» «versions » d'un «même» «texte»? Ne s'agirait-il pas plutôt de deux «textes poétiques» autonomes?

Pour Cesare Segre, le problème que posent les « rédactions multiples » est le suivant :

Au cours de son travail sur le texte (qui peut relever de la génétique ou de la variantistique) l'auteur parvient à une phase qui réalise, selon lui, une valeur définitive. Dans un second temps, l'auteur considère insatisfaisante la valeur qui a été atteinte, et moyennant une reprise du travail d'élaboration, il parvient à une valeur différente, qui correspond à la deuxième rédaction. Le lecteur se retrouve ainsi face à deux structures ou plus ; elles peuvent toutes être analysées dans leur synchronie, c'està-dire de manière statique, alors que la confrontation de deux structures le conduit à une analyse dynamique sur le plan de la diachronie. La dynamique de la poétique de l'auteur prend ainsi le relais de la dynamique de l'œuvre23.

Sur la base de cet exemple et de cette invitation à penser la variation textuelle dans les termes d'une stratégie globale, qui nous fait passer d'une «structure» à l'autre, voyons maintenant, de manière modeste, ce que peuvent nous enseigner quelques travaux émanant des traditions philologiques et éditoriales européennes (italienne et allemande) et anglo-américaine sur ce point. Précisons cependant que si, dans le cadre de la «variantistica», Cesare Segre a envisagé le problème des «rédactions multiples » qui relèvent de l'avant-texte ou du texte, je n'aborderai pour ma part cette question que dans la phase éditoriale, c'est-à-dire pour des états textuels ayant fait l'objet d'une ou plusieurs rééditions. Ce n'est qu'à partir de ce stade-ci que se pose, selon moi, la question de savoir si nous sommes face à des «versions» d'un «même» texte ou à des «textes autonomes».

23. C. Segre, «Il problema delle redazioni plurime», dans La filologia testuale e le scienze umane, Roma, Accademi Nazionale dei Lincei, 1994, p. 177-178; je traduis et souligne. 


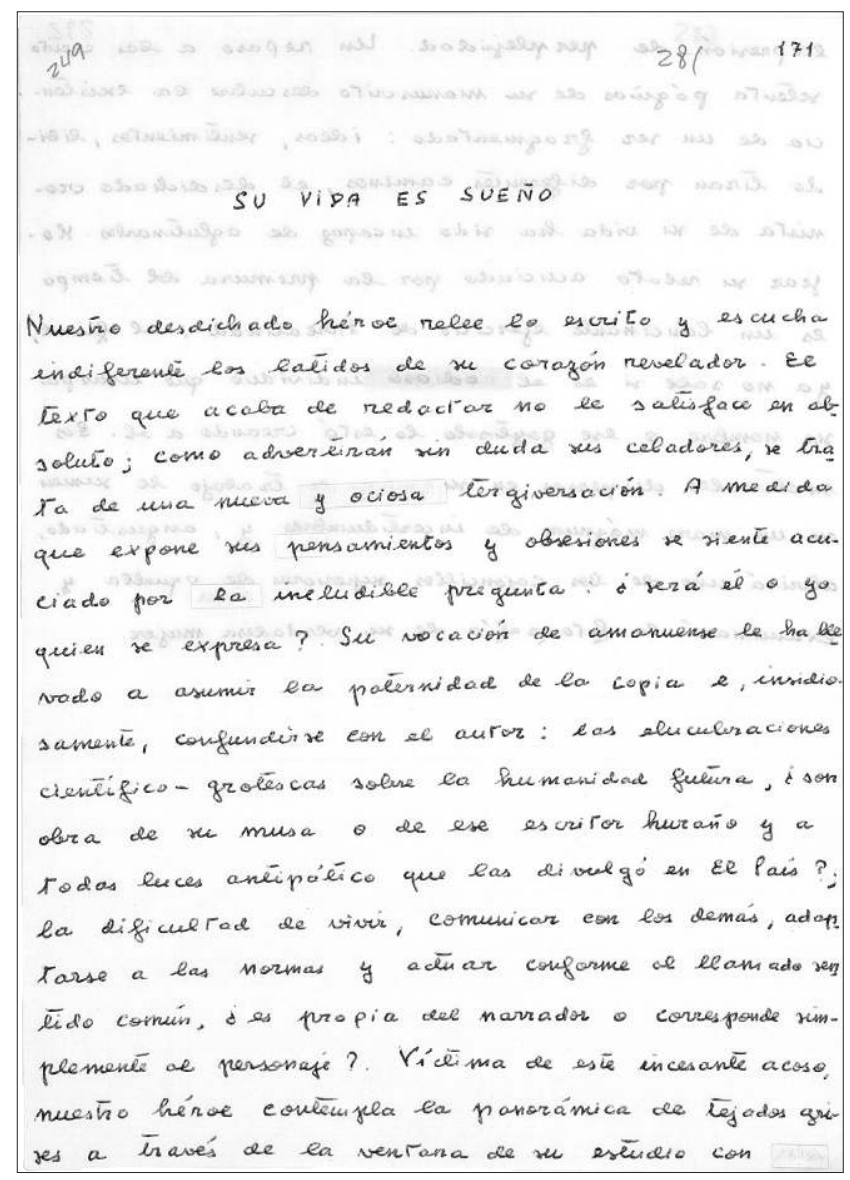

Fig. 2 : Brouillon définitif de la séquence «Su vida es sueño» (MSI 281) (C) Juan Goytisolo

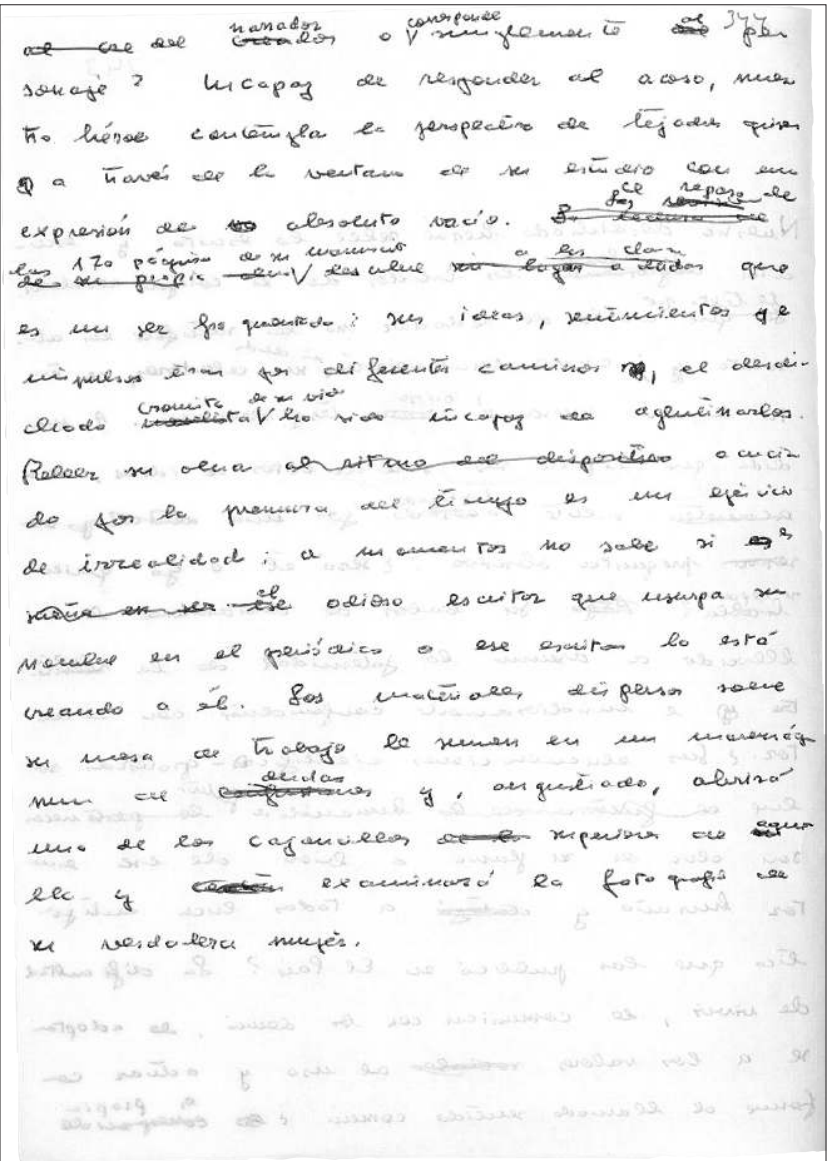

Fig. 3 : Premier brouillon de la séquence «Su vida es sueño» (MSI 344) (C) Juan Goytisolo
Les éditeurs scientifiques anglo-américains (Bornstein, Shillingsburg, Bryant, Eggert...) qui s'inscrivent dans la mouvance de la New New Bibliography présentent, ce me semble, quelque accointance avec la génétique éditoriale et la critique génétique, conçues comme étude «des processus de l'engendrement textuel». Pour Shillingsburg, à partir des années quatre-vingt, les éditeurs scientifiques anglo-américains étaient d'avis que «scholarly editions organize and present works of art primarly as process not primarly as product $24 »$. Et d'aucuns enracinent cette idée dans un article de Lebrave paru en anglais : «Rough Drafts : A Challenge to Uniformity in Editing 25 » qui permettrait de soutenir que l'étude des processus peut être étendue des brouillons aux textes 26 .

À mon sens, les éditeurs anglo-américains du texte moderne se démarqueraient toutefois de la tradition française, car, ayant fait leur le changement de paradigme auctorial de McGann et McKenzie, ils sont prêts à tirer les ultimes conséquences de la socialisation du processus

24. Peter Shillingsburg, «Author, Texts, and Polemics of Textual Criticism», dans Devils and Angels. Textual Editing and Literary Theory, dir. P. Cohen, Charlottesville/London, UP of Virginia, 1991, p. 26.

25. Text, nº 3, 1987, p. 35-42.

26. Beth Luey, Editing Documents and Texts. An Annotated Bibliography, Madison, Madison House, 1990; David C. Greetham, Textual transgressions : essays toward the construction of a biobibliography, New York, Garland, 1998; J. Bryant, op. cit.
Versioning, fluid text ou version autorisée? 
d'écriture et enclins à repousser les frontières du «texte variant » jusqu'à la phase 5 de Lebrave, qui inclut «les altérations postérieures à la dernière édition autorisée 27 ». Ainsi, ils ne substituent plus seulement à l' auteur une « instance éditeur (editor)» de caractère collaboratif, mais aussi une «fonction auteur», qui, outre les producteurs (écrivain et editor), comprend les consommateurs (lecteurs et éditeurs) ${ }^{28}$. Prenant le relais de Reiman, partisan du versioning ${ }^{29}$, de Bornstein, qui parle du livre palimpseste, de Grigely, qui a créé le néologisme textaltérité, ou de Stillinger, défenseur d'une multiple Authorship, Bryant considère qu'une fois accepté le caractère social, définitivement instable et pluraliste du texte, «the only "definitive text" is a multiplicity of texts, or rather, the fluid text» (op. cit., p. 2).

Éditeurs des œuvres de Thackerey (Shillingsburg), Coleridge (Stillinger), Byron, Rossetti (McGann), Melville (Bryant), D.H. Lawrence (Eggert), ces chercheurs ont aussi su tirer parti de la révolution numérique et ont cherché à développer des outils pour mettre en valeur cette conception du texte instable ou texte fluide. Tout comme les généticiens face aux volumineux dossiers génétiques, les partisans de la New New Bibliography ont ainsi très tôt compris que le numérique permettrait d'offrir aux lecteurs de manière dynamique toutes les «versions » d'un «texte», en mettant à mal les limites physiques du livre.

Dans The Fluid Text. A Theory of Revision and Editing for Book and Screen, John Bryant insiste sur le fait que mettre l'accent sur le texte fluide implique de renoncer au confort et au plaisir du texte stabilisé, prêt pour être lu (clear reading text). Dans le cadre d'une telle analyse, l'accent ne tombe plus sur le texte, mais sur la distance qui existe entre les textes, sur les changements (p. 123). Un objectif que l'on pourrait, d'entrée de jeu, rapprocher de celui que permet un outil comme MEDITE et qui semble bien être l'objectif premier de la génétique éditoriale, telle que la présente Mahrer dans ce volume, et le sera dans son projet de collection «Variance» (Mahrer, p. 35).

Néanmoins, si les propositions des Anglo-Américains ne sont pas dépourvues d'intérêt, notamment en termes de «pratique éditoriale», elles ne me semblent pas apporter de renouveau sur la question théorique que pose le texte variant.

Pour Bryant, en 2002, le problème du texte variant pouvait être ramené à deux questions clés : «What degree and manner of variation between two texts of the same work are required for us to label each a version of the work? What makes a version worthy of critical analysis, and hence editing? ? (op. cit., p. 70). Et pour essayer d'y répondre, il est reparti, tout comme McGann en son temps, de quelques travaux canoniques des «philologues » germanophones traduits en anglais ${ }^{30}$, à savoir, Hans Zeller, Gunter Martens et Siegfried Scheibe, qu'il range sous la bannière des «généticiens européens » (group of largely European textual scholars known as «geneticist», ibid., p. 70).

27. «Manuscrits de travail et linguistique de la production écrite», art. cité, p. 18.

28. J. Bryant, op. cit. et P. Shillingsburg, «Authority and Authorization in American Editing», art. cité.

29. D.H. Reiman (" "Versioning": The Presentation of Multiple Texts», Romantic Texts and Contexts, Columbia, University of Missouri Press, 1987, p. 167-180) entend par là «la présentation d'une multiplicité de textes » (p. 168) qui ne sont pas préparés par l'éditeur et qui permettent au lecteur de découvrir par lui-même la pluralité des états comme des « touts ». Il présente le versioning comme une alternative à l'editing (p. 169), sans pour autant considérer le travail de l'éditeur obsolète.

30. Hans Walter Gabler, George Bornstein, Gillian Borland Pierce (dir.), Contemporary German Editorial Theory, Michigan, University of Michigan, 1995. 
Or, et c'est bien ce qui nous intéresse, c'est aux mêmes auteurs et aux mêmes textes (de 1975 et 1991, notamment des actes d'un congrès tenu à Berlin en 1990 et publiés sous le titre Zu Werk und Text) qu'Alfredo Stussi renvoyait en 1994 - et dans l'édition revue de 2007 -, lorsqu'il évoquait le problème des «redazioni plurime» traité par Cesare Segre.

Confronté au texte variant, c'est au critique, selon Stussi, qu'il incombe de trancher s'il s'agit de deux versions d'un même texte ou de deux textes et donc d'opter pour une présentation texte-apparat ou pour l'édition de deux textes. Or il découle du caractère éminemment interprétatif de cette décision, que celle-ci pourra être remise en question car «confronté à une représentation synthétique du type texte-apparat critique de variantes, l'usager restera toujours libre de penser qu'il s'agit de deux entités autonomes, de deux systèmes autosuffisants ${ }^{31}$ ».

Éditeur des œuvres de Conrad Ferdinand Meyer, Hans Zeller avait, pour sa part, et dans un premier temps, essayé de résoudre le problème version-texte de manière technique et objective, en se basant sur les définitions de la version (Fassung) et de la variante (Variante) de Siegfried Scheibe, éditeur des œuvres de Goethe, dont les réflexions théoriques sur l'édition de texte firent autorité en Allemagne jusque dans les années quatre-vingt-dix ${ }^{32}$. Ces définitions ont, depuis lors, été remises en question par Reuß, tout d'abord, puis par Zeller et Martens.

Pas plus ici que dans le cas des philologues éditeurs anglo-américains, je ne puis rentrer dans le détail d'un débat polémique qui dépasse les objectifs de cet article. Contentons-nous ici de signaler que, en questionnant implicitement toute approche essentialiste de la version (Fassung), Stussi rejoint en réalité, sans le savoir, Roland Reuß qui, en 1990, dans un article passionnant, mais trop rarement cité, a critiqué sévèrement l'approche de Zeller-Scheibe débattue à Berlin la même année ${ }^{33}$. Comme il était rappelé dans notre citation épigraphe, la version n'est qu' un concept descriptif.

Une «version» n'a pas d'existence objective, elle n'est pas là en tant que telle. Un fragment langagier ne peut être dit «version» que grâce à un éditeur [scientifique]. Une «version » est donc un concept descriptif, et non pas un concept qui se définit par ce qu'il est en et par lui-même34.

Autrement dit, il n'y a pas de version en soi, c'est au critique de désigner (nennen) ce qu'est une version, ce qu'est un texte et d'expliciter les raisons pour lesquelles deux éditions - dans ce cas, de «Michael Kohlhaas» (1808) et Michael Kohlhaas (1810) - généralement présentées comme deux «versions » d'un texte peuvent être considérées comme deux textes. Mais, par la suite, Reuß montre également pourquoi la simple «désignation» - version, texte - fait plus que décrire. En réalité, elle in-forme - parfois dé-forme - l'objet d'étude en présupposant résolue la question de l'identité textuelle (Textidentität) (p. 11-14).

31. Alfredo Stussi, Introduzione agli studi di filologia italiana, Bologna, Il Mulino, 2007 [1994], p. 162-163; je traduis et souligne.

32. Avec de légères modifications, la définition de la version se retrouve depuis les années soixante dans différents articles de Scheibe visant à asseoir la terminologie et les fondements de la textologie (Siegfried Scheibe, Kleine Shriften zur Editionswissenschaft, Berlin, Weidler, 1997).

33. La réflexion de Reuß sur la «version» se trouve au fondement de l'édition de Valle-Inclán à paraître (Vauthier et Santos Zas, Ramón del Valle-Inclán. Un día de guerra..., op. cit.). Elle est également à la source d'un récent travail de Janina Reibold sur Hamann («Philological Challenges of Hamann's Fliegender Brief», dans The Future of Philology, dir. Hannes Bajorh et al., Cambridge, Cambridge Scholars Publishing, 2014, p. 98-119).

34. Roland Reuß, «"Michael Kohlhaas" und "Michael Kohlhaas". Zwei deutsche Texte, eine Konjektur und das Stigma der Kunst», Berliner Kleist Blätter, 3, 1990, p. 6; je traduis. 
Quelques années plus tard, soit en 1998, Reuß a pris note de la seconde tentative de Zeller 35 de définir la version en renonçant au critère d'identité textuelle (Textidentität), emprunté à Scheibe, au profit d'une similitude de motif (Motiv), mais il lui reproche toujours d'escamoter le seul critère fondé en raison pour justifier ses choix : le jugement critique.

En 2003, et en dépit des attaques dont il avait fait l'objet, Hans Zeller, qui n'était pas seulement un théoricien hors pair, mais aussi beau joueur, donnera raison à Reuß. Ainsi, dans son bilan sur «Le développement de l'édition génétique au $\mathrm{XX}^{\mathrm{e}}$ siècle » il déclare :

Comme on l'a vu, il n'existe pas de définition généralement praticable du concept de version. Il revient à l'éditeur de décider de manière souveraine «s'il doit considérer deux textes ou plus, comme des versions d'une œuvre ou comme différentes œuvres ». Il n’y a que des décisions pragmatiques au sujet de la frontière qui existe entre différentes versions d'une œuvre et différentes œuvres 36 .

Et il renvoie au travail de Reuß qu'il cite en note de bas de page. Loin de constituer une exception, l'impossibilité de donner une définition univoque et objective de la «version», touche d'autres concepts clés de l'édition pour lesquels les philologues allemands semblent aujourd'hui renoncer à l'idée d'atteindre un consensus (tels « œuvre», «texte», «variante»).

Ce bref aperçu d'un débat, dont les principaux jalons se sont échelonnés pendant quinze ans (1990-2003) et qui débouche, on le voit, sur l'aveu d'un échec terminologique : «il n'existe pas de définition généralement praticable du concept de version », explique, à mon sens, pourquoi le souhait de certains 37 d'établir les bases d'un «Dictionnaire de philologie éditoriale» semble aujourd' hui en recul par rapport à l'appel (plus modeste) à la cohérence et à la rigueur terminologique. Ce qui implique, notamment, comme le montre Nutt-Kofoth, qu' on ne perde pas de vue que les concepts ont une histoire et une réception transnationale ${ }^{38}$. La réception décalée des concepts allemands de «version» ou de «texte» chez Bryant - et indirectement chez Stussi, car la réédition de cette partie de son manuel n'est pas actualisée - en est une illustration. En effet, tous deux se réfèrent à des travaux des années soixante-dix et quatrevingt-dix, depuis longtemps remis en question en Allemagne.

Pour nous en tenir maintenant au contexte germanique, on ne peut comprendre pourquoi la question des relations entre «variante», «version» (Fassung), «texte» a mobilisé toute l'énergie des philologues allemands depuis les années soixante-dix si on ne se rappelle pas que cette question était liée à celle de «l'intention» - la fameuse «volonté de l'auteur» - et à la méthode devant présider au choix et à l'établissement d'un texte dit «de référence» (copy-text, dernière version, princeps ou tout simplement «version autorisée»). La problématique est centrale - notamment pour les éditeurs - et Hans Zeller l'aborda à deux reprises dans des

35. Hans Zeller et Jelka Schilt, «Werk oder Fassung eines Werks? Zum Problem der Werkdefinition nach Fassungen am Beispiel von Conrad Ferdinand Meyers Gedichten», dans Zu Werk und Text. Beiträge zur Textologie (Hsg. Siegried Scheibe, Christel Laufer), Berlin Akademie Verlag, 1991, p. 61-86.

36. H. Zeller, «Die Entwicklung der textgenetischen Edition im 20. Jahrhundert», dans Geschichte der Editionsverfahren vom Altertum bis zur Gegenwart im Überblick (Hsg. R. Nutt-Kofoth), Tübingen, Max Niemeyer Verlag, 2003, p. 145 ; je traduis.

37. Günter Martens (dir.), Editorische Begrifflichkeit. Überlegungen und Materialen zu einem "Wörterbuch der Editionsphilologie”, Editio, n 36, 2013.

38. Rüdiger Nutt-Kofoth, «Editorische Axiome», Editio, n² 26, 2012, p. 59-71. 
articles confrontant les traditions anglo-américaine et allemande sur ce point ${ }^{39}$. Ainsi, pendant que les éditeurs anglo-américains ont cherché à atteindre de manière surannée une prétendue volonté de l'auteur, les germanistes ont préféré déplacer les termes du débat sur le terrain de $l^{\prime}$ « autorisation 40 ».

La notion de «version autorisée » devait d'abord permettre de résoudre la question d'une possible préséance éditoriale : pour l'éditeur historien (qui prend le relais d'un éditeur «exécuteur testamentaire»), il n'y a plus de «bonne» ou de «mauvaise» version, encore moins de «meilleure» version mais, tout au plus, différentes versions (synchronie) qui se succèdent dans le temps (diachronie). Chacune d'entre elles a donc sa légitimité historique. Idéalement, la documentation devrait être reproduite de manière exhaustive, et sans intervention éditoriale aucune. Sous la pression des circonstances, et pour des raisons de volume, l'éditeur est souvent conduit à choisir un texte plutôt qu'un autre et à construire un apparat historico-critique qui doit respecter les principes de complétude définis, c'est-à-dire exposer toute l'histoire du texte. D'autre part, parler de version «autorisée », c'était aussi reconnaître que l'œuvre d'art, sitôt sortie de l'atelier de l'écrivain, entrait dans un champ de forces sociales dont il pourrait être vain, par la suite, de chercher à démêler les influences. Faisant sienne la conclusion de James Thorpe, l'un des détracteurs de l'école Greg-Bowers de la première vague, Zeller écrit " "the integrity of the work of art" include "those intentions which are the author's, together with those others of which he approves or in which he acquiesces" 41 ». Cette déclaration est capitale, car elle implique que l'éditeur critique n'a plus le droit d'intervenir dans le texte, en prétendant séparer le bon grain auctorial de l'ivraie éditoriale. Dans une optique d'ascendance structuraliste, parler de «version autorisée » signifie que celle-ci, dans son ensemble ou comme tout, l'a été par l'auteur (nous retrouverions ici la valeur du bon à tirer), même s'il peut arriver que dans des cas résiduels et sous des conditions strictes elle puisse se trouver suspendue. Dans ce cas et dans ce cas seulement, on parlera de «faute» (Textfehler) et l'éditeur pourra intervenir. L'emendatio ne sera toutefois recevable que si la correction est sans équivoque. Et même dans ce cas-là, elle sera reportée dans la note de bas de page, ce qui permettra d'éviter la création de «diasystèmes 42 ». Plus question, donc, de corriger des erreurs de contenu, de

39. H. Zeller, «A new Approach to the Critical Constitution of the Literary Texts », Studies of Bibliography, $\mathrm{n}^{\circ} 28$, 1975, p. 231-264; «Struktur und Genese in der Editorik. Zur germanistischen und anglistischen Editionsforschung », LiLi. Zeitschrifit für Literaturwissenschaft und Linguistik, 5, Heft 19/20, 1975, p. 105-126.

40. Remarquons que ce déplacement ne résout pas entièrement le problème, et que ce débat reste l'un des plus polémiques dans le domaine éditorial. Dans son article «The Concept of Autorisation» (Text, $\mathrm{n}^{\circ}$ 14, 2002, p. 77-90), Marita Mathijsen souligne d'entrée de jeu que «Not only do anglo-American and German views on "autorisation" diverge, but the most prominent theorists have considerably modified their view in the last few years» (p. 77) et conclut en affirmant que «it will be difficult to achieve an internationally accepted definition of the concept of autorisation» (p. 90). Deux ans plus tard, Shillingsburg fait montre du même scepticisme, comparant ce débat à l'histoire de la tentation dans le jardin d'Éden («Authority and Authorization in American Editing», art. cité, p. 73-81). 41. James Thorpe cité par Hans Zeller, «A new Approach to the Critical Constitution... », art. cité, p. 38. Dans les lignes qui suivent, j'essaie de présenter quelques temps forts de cet article, ainsi que de «Struktur und Genese in der Editorik. Zur germanistischen und anglistischen Editionsforschung» (art. cité), publié la même année par Zeller 42. Cesare Segre utilise ce concept pour mettre à jour les interférences produites lors de la collusion entre le système de l'auteur et celui du copiste. Nous l'utilisons ici de manière figurée pour évoquer la pratique éditoriale moderne qui généralement consiste à corriger à même le texte, de manière posthume ou anthume, ce qui débouche inévitablement sur de nouveaux textes. Voir C. Segre, «Critique textuelle, théorie des ensembles et diasystèmes », Bulletin de la classe des lettres et des sciences morales et politiques de l'Académie de Belgique, $\mathrm{n}^{\circ}$ 62, 1976, 
possibles coquilles ou encore des soi-disant interventions éditoriales... sous prétexte qu'elles n'ont pas été «voulues par l'auteur». «J'espère pouvoir montrer, écrit Martens en 2005, que la "retenue éditoriale", la "non-intervention", peut révéler une conscience critique bien supérieure à la témérité correctrice de certains éditeurs» (p. 11; je traduis). Forte de ces éléments, nous pouvons revenir au cas de figure présenté dans notre apparente digression sur Paysages après la bataille et conclure.

\section{Conclusion}

Chercher à savoir ce que les traditions philologiques allemande, italienne et angloaméricaine du «texte moderne» peuvent nous apprendre sur la manière de traiter les «transformations effectuées par l'auteur après la mise en circulation du texte » c'est reconnaître que cette question a intéressé et intéresse encore les philologues qui cherchent à éditer ou, plus exactement même, à rééditer des textes imprimés, dont il existe très souvent plusieurs éditions. Pour des raisons bien connues, les pionniers de la critique génétique ont, au cours des années de fondation et d'institutionnalisation de leur discipline (1975-1990), cherché à dessiner les contours de leur objet (le manuscrit moderne) et à définir leurs objectifs (l'étude du processus de création); ils l'ont notamment fait par opposition, à ce qui se faisait dans d'autres domaines de la recherche littéraire en France, mais aussi, par opposition au travail des philologues et éditeurs allemands, qui, à l'époque, s'intéressaient prioritairement au texte édité et à éditer - plutôt qu'aux manuscrits 43 . Au cours du premier lustre des années quatre-vingt-dix, les généticiens ont élargi les frontières de leur champ de recherche pour aller à la rencontre non plus des seuls philologues et éditeurs allemands, mais aussi de leurs homologues italiens et anglo-américains - sans même parler du projet international de littérature hispano-américaine Archivos ${ }^{44}$.

p. 279-292 et «Les transcriptions en tant que diasystèmes », «La pratique des ordinateurs dans la critique des textes », colloques internationaux du CNRS, Paris, CNRS, 1979, p. 45-49.

43. Dans son article précédemment cité, Nutt-Kofoth rappelle ainsi que dès la fin des années soixante, puis dans les années quatre-vingt-dix, la critique génétique française a toujours cherché à se démarquer clairement des sciences de l'édition critique (Editionswissenschaft) allemande («hat sich immer deutlich von der germanistischen Editionswissenschaft abgegrenzt»). Selon lui, la «maxime axiomatique» de cette démarcation se trouve dans une formulation de Grésillon, pour qui «La critique génétique interprète les manuscrits, les sciences de l'édition critique (Editionswissenschaft) éditent les manuscrits» (p. 67). Et le philologue allemand renvoie notamment au passage de l'article de Grésillon «Bemerkungen zur französischen "édition génétique" (1998), dans lequel la généticienne montre tout à la fois la différence et la complémentarité entre les organes de diffusion les plus représentatifs des deux traditions : Genesis et Editio (p. 67). Selon Nutt-Kofoth, l'opposition de Grésillon ne tient pas compte des développements des sciences de l'édition critique allemande depuis les années quatre-vingt-dix. Il suggère ensuite de lire cette démarcation axiomatique à la lumière d'une orientation interne à la France («innerfranzösische Zielrichtung») (p. 68). Grésillon, pour sa part, commence son article en soulignant que bien que proches voisins, les Français et les Allemands n'ont pas toujours pris la peine de discuter les théories et pratiques éditoriales issues de l'autre côté du Rhin. Bien plus, «à quelques exceptions louables près, la France continue d'ignorer la tradition allemande de l'édition de texte scientifique ( «Frankreich, von einigen löblichen Ausnahmen abgesehen, ignoriert weiterhin die deutsche Tradition der wissenschaftlichen Textedition...») (p. 52).

44. B. Vauthier, «¿Crítica textual? ¿Critique génétique? ¿Filologia d'autore? ¿Crítica genética?...», art. cité et «¿Critique génétique y/o filologia d'autore? Según los casos...», art. cité. 
Avec le recul, et même s'il existe de notables exceptions, ces mêmes années semblent pourtant avoir été également celles de la fin du dialogue ${ }^{45}$. Les généticiens français se sont progressivement repliés sur leurs terres et leur objet - l'avant-texte - qu'ils ont plus souvent étudié et commenté qu'édité, et surtout ils ont cantonné leurs interlocuteurs philologues de l'autre côté de la frontière, dans le champ de ce texte dit «clos»-bien qu'il soit, on vient de le voir, très dynamique. La rupture - dont traite l'article de Lebrave «La critique génétique : une discipline nouvelle ou un avatar moderne de la philologie?»- est caduque, puisque dès 1995 des germanistes de renom (Zeller, Martens, Reuß) ont fait ouvertement leurs les principes d'édition des manuscrits de travail des généticiens français 46 . Mais les critiques non germanophones ignorent encore trop souvent que l'état de l'art philologique dont parle Lebrave en 1992 appartient désormais à l'histoire de la discipline.

Un rapprochement ou une réconciliation des deux disciplines à travers la «poétique de transitions entre états » est sans conteste un événement appelé à faire date dans le domaine francophone, si on considère que ce sont les généticiens qui, cette fois, semblent disposés à traverser la frontière. Mais pour que cette rencontre puisse être fructueuse et ne se fasse pas à contretemps, comme semble l'être en partie celle de Bryant, il nous faut réellement prendre acte, et plus encore connaissance, des travaux - nombreux en la matière et passionnants - sur le «texte moderne» des philologues allemands, italiens et anglo-américains disponibles dans ce domaine, les Espagnols y faisant, par contre, figure de parents pauvres ${ }^{47}$.

Sur la base des quelques jalons exposés ci-dessus, ce sont, à mon sens, les travaux théoriques des sémioticiens et des philologues italiens et allemands qui nous aideront le mieux à affronter les «difficultés épistémologiques que soulève ce problème de textes à la fois identiques et différents 48 ». Que ce soit avec Cesare Segre, Alfredo Stussi, Roland Reuß ou Hans Zeller, nous pouvons affirmer qu'il n'y a pas de «version en soi» : c'est au critique qu'il revient de déterminer ce qu'il considère comme «versions d'un texte» et ce qu'il considère comme «textes autonomes». Cette décision ne sera, certes, pas sans conséquences sur la suite du projet (éditorial) car sitôt qu'il choisit de parler de deux «versions »- plutôt que de deux «textes»-le critique pose (et non suppose) une identité textuelle, qui est affirmée par-delà les différences qui séparent les objets. La décision justifiera probablement l'idée de collation des séquences textuelles, ou à l'inverse permettra de défendre l'idée du «dynamisme de la poétique de l'auteur», qui, dans le cas de l'édition de Goytisolo, justifierait la publication

45. Pour une première mise au point sur cette question, on lira l'article de Geert Lernout, «"Critique génétique" und Philologie», dans Text und Edition. Positionen und Perspektiven, Berlin, Erich Schmidt, 2000, p. 121-142. En ce qui concerne les exceptions, il suffit de penser à la participation régulière d'Almuth Grésillon aux rencontres des germanistes et à ses publications en allemand, par exemple, dans Editio.

46. H. Zeller, «Die Fasksimile-Edition als Grundlagenedition für Philologie und Textgenetik. Ein Vorschlag». Textgenetische Edition. Beihefte zu Editio, ${ }^{\circ} 10,1998$, p. 80-91. Je ne reviendrai pas ici sur le fait que l'article de Lebrave est également une réponse implicite aux attaques dont les généticiens avaient fait l'objet dans le chef de leurs collègues. Ainsi quand Lebrave parle de Cousin, Albalat et Lanson comme de «faux » précurseurs, il le fait en référence à ceux qui, en France, les avaient accusés de ne pas reconnaître leurs dettes (B. Vauthier, «¿Crítica textual? ¿Critique génétique?...», art. cité, p. $49 s q$ ). Pour des dissensions internes plus tardives, on consultera également l'article de G. Lernout, précédemment cité. Et Nutt-Kofoth, art. cité, p. 68, n. 26. Lebrave est également revenu sur ses déclarations lors d'une conférence prononcée à Salvador de Bahía (1999), encore inédite.

47. B. Vauthier, M. Santos Zas (éd.), Un día de guerra..., op. cit.

48. J.-G. Ganascia, J.-L. Lebrave, art. cité, p. 74. 
intégrale de deux, voire trois textes, à savoir, la princeps de 1982, l'édition de référence illustrée de 1987 et l'édition revue (et raccourcie) de 2006. Ces trois éditions permettraient en effet de valoriser non seulement la dimension proprement textuelle ou sémantique du «texte», mais aussi sa dimension matérielle (material bibliography), une dimension que les éditeurs anglo-américains ont, à mon sens, valorisée jusqu'à présent mieux que tout autre et qui pourrait être rapprochée de l'attention que les généticiens accordent à la dimension sémiotique du manuscrit.

Pour Reuß, le plus radical dans ses positions parmi les germanistes, soutenir que nous sommes face à deux textes n'implique pas seulement qu'ils doivent être publiés de manière autonome. Cela signifie aussi qu'on renonce à une présentation en parallèle ou même à la constitution d'un apparat critique. Face à deux «textes poétiques», il n'y pas d'identité textuelle qui justifie la juxtaposition. Et la constitution d'un apparat critique pour des raisons pragmatiques ne change rien au statut «poétique » - au sens structuraliste - du texte. L'apparat est et restera un compromis qui se fait toujours au détriment de l'individuel - et du savoir linguistique, esthétique, historique -, et en faveur de l'ergonomie de la lecture. Sa justification reste au mieux que sans lui certains textes ne seraient peut-être pas transmis du tout 49 .

Parler de version ou de texte «autorisé» a, d'une part, permis aux Allemands d'intégrer très tôt la dimension sociale ou collaborative de l'auctorialité du texte, tout en la restreignant implicitement à la durée de vie de l'auteur. D'autre part, cette notion les a aidés à résoudre de manière pragmatique le problème de la granularité de la variation textuelle, puisque, si en théorie une seule altération (ponctuation, orthographique, syntaxique, etc.) suffit pour établir une variation entre deux unités textuelles, dans le cas d'un texte imprimé, on parlera de version ou de texte «autorisé», et donc «authentique», quand il est établi que l'auteur a participé au processus de réécriture ou approuvé le résultat final50. L'autorisation vaut pour ce «tout» structuré. Dans la lignée de ce qu'affirme Segre au sujet des «états textuels ou valeurs successivement atteints», Roland Reuß considère ainsi que «si un texte poétique est autorisé, nous n'avons aucune raison de croire que l'idée qui y est développée n'y est pas réalisée complètement ${ }^{51}$ ». Mais, à la différence de Segre et des philologues italiens, Reuß s'en tient toutefois sur ce point aux seuls états textuels stricto sensu, et ne cherche pas à les rapprocher des campagnes avant-textuelles. Ce qui nous permet de préciser que si les éditeurs allemands prennent en considération la totalité du processus d'écriture - des phases 1 à 4 - dans le cadre d'éditions dites historico-critiques - qui sont aussi génétiques -, ils souhaitent maintenir, Reuß en tête (2005), une différence de principe entre avant-texte et texte. La frontière doit notamment servir de garde-fou pour éviter que l'éditeur ne redevienne ce «co-auteur» qui sait ou croit savoir ce que voulait l'auteur. Car confronté à des incongruités textuelles, il est souvent tenté de recourir à des matériaux auctoriaux et autographes pour conforter ses interventions dans le corps même du texte, celles-là même qui sont souvent à l'origine de nouveaux «textes», de nouvelles versions. (Je pense qu'il n'est plus guère besoin d'insister ici sur le fait que mon intervention ponctuelle dans le corps du texte autorisé par Juan Goytisolo tombe sous le coup de cette observation.)

49. R. Reuß, «"Michael Kohlhaas” und "Michael Kohlhaas”...», art. cité, p. 9-15.

50. H. Zeller, «Die Entwicklung der textgenetischen Edition im 20. Jahrhundert», art. cité, p. 178.

51. R. Reuß, «"Michael Kohlhaas" und "Michael Kohlhaas"...», art. cité, p. 8. 
Ces nouvelles versions, ces nouveaux textes sont justement ceux qu'assument, que revendiquent même les philologues anglo-américains du texte moderne. Car comme je l'ai évoqué plus haut, dans le cadre d'une approche résolument processuelle de la création littéraire, ils considèrent que la socialisation de la création ne s'arrête pas à la mort de l'auteur. L'idée d'autorisation n'est pour eux qu'un pis-aller ou un subterfuge qui contourne le problème de l'intentionnalité sans le résoudre. Ils préfèrent y renoncer au profit de celui de fluidité du texte qui oblige d'étendre le domaine de la socialisation «beyond the writer's death and "will" 52 ».

Bien que cette option ne soit pas la mienne, vu qu'elle conduit à un élargissement de l'étude des processus aux «altérations postérieures à la dernière édition autorisée 53 », il faut reconnaître qu'elle a le mérite de rejoindre l'expérience du lecteur ordinaire, qui découvre souvent les classiques de la littérature même moderne - le canon - sous une forme et dans un format (édition ou réédition) qui peut malmener à ce point un original auctorial, qu'ils justifieraient le qualificatif d'apocryphe ${ }^{54}$. 
BÉNÉDICTE VAUTHIER est professeure ordinaire de littérature espagnole à l'Université de Berne et directrice de l'Institut de langue et littératures hispaniques. Elle a travaillé à l'Université de Cáceres, Autonome de Madrid, Liège, Bonn et Tours. Ses recherches portent sur la littérature espagnole contemporaine, la théorie de la littérature, notamment, de l'édition, et sur l'étude et l'édition de manuscrits et textes hispaniques modernes (XXe-XXI e siècles). Elle a publié une quinzaine de monographies (sur Menéndez y Pelayo, Unamuno, Juan Goytisolo), traductions (Unamuno, Ortega y Gasset) et éditions (Unamuno, Guillén, Goytisolo, Valle-Inclán).

benedicte.vauthier@rom.unibe.ch

\section{Résumés \\ Éditer des états textuels variants}

A près avoir fait retour sur un cas concret d'édition, sont examinés ici quelques problèmes théoriques que soulève la pratique d'éditeur scientifique quand elle aborde le «texte» publié dans sa dimension processuelle. Il en va ainsi du choix de l'édition (quel état textuel privilégier?), de l'auctorialité multiple (qui corrige ?), des modes d'intervention éditoriale mais aussi et surtout des rapports entre version et texte dans la phase éditoriale (qu'éditer? que représenter et comment?). Poursuivant une recherche de caractère comparatiste, on examine comment la génétique éditoriale française entre en dialogue avec les traditions philologiques européennes et anglo-américaines du texte moderne qui font une place à l'étude des «textes variants », problème qui est identifié comme étant celui des «redazioni plurime» (Cesare Segre) et circonscrit ici à la phase éditoriale. Dans la conclusion, on salue la mise en place d'une «poétique de transitions entre états » (Lebrave) et on dégage les atouts d'une réflexion théorique sur l'édition de textes de caractère international.

After considering a concrete editorial case, we will examine a few theoretic problems raised by the praxis of a scientific publisher when it approaches the published "text" in its procedural dimension. The same applies to the choice of the edition (which textual phase should be privileged?), of the multiple auctoriality (that corrects?), the editorial intervention modes and particularly the connections between text and version in the editorial phase (what is editing? represent what and how?). Continuing a research with a comparatist approach, we will examine how French editorial Genetics dialog with European and Anglo-American philological traditions of the modern text which include the studies of "variant texts", a problem identified as that of the "redazioni plurime" (Cesare Segre) and circumscribed here to the editorial phase. To conclude, we welcome the setting up of a "poetics of transitions between phases" (Lebrave) and bring to light the assets of a theoretical reflection on the edition of international texts.

Nachdem auf eine Edition Bezug genommen wurde, werden hier einige theoretische Probleme untersucht, die in der Praxis eines wissenschaftlichen Editors vorkommen, wenn sie an den publizierten „Text“ in seiner prozessualen Dimension herangeht. Es geht dabei um die Auswahl (welcher Textzustand ist zu bevorzugen), um mehrfache Urheberschaft (wer korrigiert?), um Arten der Intervention, aber auch und vor allem um Beziehungen zwischen Version und herausgegebenem Text (was herausgegeben? was wie darstellen?). Im Rahmen einer komparatistischen Untersuchung studieren wir, wie die französische critique génétique in einen Dialog tritt mit den europäischen und angelsächsischen philologischen Traditionen des modernen Texts, die beim Studium variierender Texte eine Rolle spielen - ein Problem, das seit Cesare Segre als redazioni plurime bekannt ist und das hier die Editionsphase umschreibt. Abschließend begrüßen wir die Einführung einer ,poétique de transitions entre états" (Lebrave) und wir unterstreichen die Vorteile einer theoretischen Reflexion über das Edieren von Texten auf internationaler Ebene.
Después de volver sobre un caso específico de edición, se examinan algunos problemas teóricos que plantea la edición científica cuando aborda el "texto publicado" como "texto en proceso". Entre aquellos, se puede mencionar la cuestión de la edición (¿qué estado del texto se ha de privilegiar?), de la autorialidad múltiple (¿quién corrige ?), de las formas de intervención editorial o, finalmente, de las relaciones entre versión y texto en la fase editorial (¿qué editar?, ¿qué representar y cómo ?). Dando seguimiento a una investigación comparatista, se examina cómo la genética editorial francesa puede entrar en diálogo con las tradiciones filológicas europeas y anglo-americanas del texto moderna que contemplan los "textos variantes", un problema que se relaciona con la cuestión de las "redazioni plurime" (Cesare Segre), pero circunscrito aquí a la sola fase editorial. En la conclusión, se alaba la puesta en marcha de una "poética de transición entre estados" (Lebrave) y se realzan las ventajas que comporta una reflexión teórica sobre la edición de textos de carácter internacional.

A partir de um caso concreto da edição, são revistos aqui alguns problemas teóricos postos pela prática do editor científico que aborda o "texto" publicado na sua dimensão processual. Estão em causa a escolha da edição (que estado textual preferir?), a pluralidade das autoridades (quem corrige?), os modos de intervenção editorial ; mas também e especialmente a relação entre texto e versão na fase editorial (que editar? que representar e como?). Prosseguindo uma pesquisa de caráter comparatista, examina-se como a genética editorial francesa entra em diálogo com as tradições filológicas europeias e angloamericanas do texto moderno, que dão lugar ao estudo dos "textos variantes", que tem afinidades com as "redazioni plurime" (Cesare Segre), aqui circunscrito à fase editorial. Em conclusão, saúda-se o advento de uma "poética das transições entre estados" (Lebrave), no quadro de uma reflexão teórica de carácter internacional sobre a edição textual.

Dopo aver affrontato un caso concreto di edizione, l'articolo esamina alcuni problemi teorici sollevati dalla pratica dell'edizione scientifica di un "testo" pubblicato, nella sua dimensione processuale. È il caso della scelta dell'edizione (quale versione del testo privilegiare?), dell'autorialità molteplice (chi corregge?), delle modalità di intervento editoriale ma anche e soprattutto dei rapporti tra versione e testo nella fase editoriale (cosa editare? Cosa rappresentare e in che modo?). Continuando una ricerca a carattere comparatista, l'articolo analizza il dialogo della genetica editoriale francese con le tradizioni filologiche europee e anglo-americane del testo moderno, che riservano un posto importante allo studio dei "testi varianti" - problema che si identifica con quello delle "redazioni plurime" (Cesare Segre) e che è qui circoscritto alla fase editoriale. In conclusione, si saluta l'avvio di una "poetica delle transizioni fra versioni" (Lebrave) e si mettono in luce i vantaggi di una riflessione teorica di carattere internazionale sull'edizione dei testi. 